\title{
SISTEMA DE VOTACIÓN PREFERENCIAL. EFECTOS EN LA COMPETENCIA INTERNA DE LOS PARTIDOS Y EN EL COMPORTAMIENTO ELECTORAL ${ }^{1}$
}

\author{
GIANLUCA PASSARELLI ${ }^{2}$ \\ Profesor de Ciencia Política \\ Universidad de la Sapienza
}

TRC, núm. 45, 2020, pp. 207-231

ISSN 1139-5583

Los sistemas electorales constituyen una materia ampliamente debatida y analizada en la doctrina. En las últimas tres o cuatro décadas se ha experimentado un gran avance en sus estudios, tanto en el plano teórico como en el empírico. Sin embargo, aun habiendo experimentado el estudio comparado de sistemas electorales un enorme desarrollo, todavía siguen apareciendo nuevos desafíos en la actualidad $^{3}$. Una definición general caracteriza a los sistemas electorales como «un conjunto de normas y de reglas de juego que regulan la competencia electoral entre los partidos y dentro de estos mismos» ${ }^{4}$. Concretamente, esta dimensión interna es la que todavía requiere de un mayor desarrollo. Aunque han aparecido algunas investigaciones y estudios de casos concretos, «la ausencia parcial de datos comparativos básicos en su versión intra partidos dificulta el desarrollo y la comprobación de esta teoría» ${ }^{5}$. Este trabajo pretende arrojar algo de luz en este

1 Traducción realizada por Gaspar González Represa (Ricercatore della Università della Campania Luigi Vanvitelli).

2 Un trabajo más extenso del autor sobre esta materia puede verse en Preferential Voting Systems. Influence on Intra-Party Competition and Voting Behaviour, Palgrave McMillan, 2019.

3 SHUGART, «Comparative electoral systems research: The maturation of a field and new challenges ahead», en Gallager y Mitchell (eds.), The politics of electoral systems, Oxford, 2005, p. 25.

4 Cox, Making votes count, Cambridge, 1997, p. 38. La cursiva es nuestra.

5 SHUGART, «Comparative electoral systems research: The maturation of a field and new challenges ahead», cit., 25. De este modo, si es cierto lo dicho por Katz y Bardi acerca de que el voto preferencial ha recibido menos atención de lo que merece, y que hasta el momento solo unos pocos autores han dedicado una investigación empírica y meticulosa sobre el asunto, este trabajo pretende de forma ambiciosa poner fin a esa ausencia. No hay duda de que en los veinte años transcurridos desde el análisis de Katz y Bardi, 
tema, explorando los efectos de un «sistema de votación preferencial», en particular, para los PLPR, la dimensión intra partidos, así como enmarcar las reglas electorales en las bases de cada sistema.

Teniendo en cuenta el comportamiento de los votantes y su influencia en los resultados electorales, podemos considerar dos grandes categorías de sistemas de votación preferencial; cuando los votantes votan directamente a una lista, o cuando no lo hacen. El proceso de asignación de escaños a cada candidatura puede ser «directo» o «mediado» por un segundo paso. En los sistemas de representación proporcional, en unos casos son los propios partidos los que establecen el orden de los candidatos en las listas y por cada escaño que obtienen asignan posteriormente al candidato correspondiente siguiendo el orden de la lista. No obstante, el sistema de representación proporcional cerrado (RPC, CLPR) no es la única opción. Hay otros sistemas que permiten al votante emitir un voto para su candidato favorito tanto de la lista de un solo partido de su elección como de varias listas de diferentes partidos. Lo relevante aquí es que los votantes deciden (total o parcialmente, de acuerdo con varios requisitos que detallaré más adelante) a quien eligen de la lista del partido. Para conseguir esto existen sistemas de lista abierta y de lista flexible. Sin embargo, como se ha dicho anteriormente, entre los sistemas electorales, la votación preferencial es un aspecto de estos al que los autores aluden a menudo, pero que carece de un adecuado y completo análisis. Es cierto que recientemente se ha producido un incremento de los trabajos científicos relacionados con las «preferencias», este no es el caso sin duda de los estudios sistemáticos y comparativos de los sistemas de votación preferencial. Se carece de una investigación que incluya todos los supuestos (o al menos los más significativos), o que vaya más allá de las meras descripciones de tecnicismos electorales, así como que se centre más en los efectos que el voto preferencial acarrea. Concretamente, se requiere una mayor atención a las consecuencias que este voto preferencial puede suponer para la organización de los propios partidos y su competición interna. De este modo, este estudio se referirá principalmente al impacto del voto preferencial en el plano interno de los partidos, cuyos datos aún se desconocen. Lejos de optar por

\footnotetext{
varios estudios y autores se han dedicado al voto preferencial en distintos países (KATZ, «Intraparty Preference Voting», en Grofman y Lijphart (eds.), Electoral Laws and their Political Consequences, New York, 1986, pp. 85-103; KARVONEN, «Preferential Voting: Incidence and Effects», International Political Science Review, vol. 25, n. 2, 2004, pp. 203-226; «Preferential Vote in Party List», en Colomer (ed.), Personal Representation: The neglected dimension of electoral systems, Colchester, 2011, pp. 119-134; MARSH, «The Voters Decide? Preferential Voting in European List Systems», European Journal of Political Research, vol. 13, 4, 1985, pp. 365-378.; ORTEGA, Los sistemas de voto preferencial. Un estudio de 16 democracias: reflexiones sobre el sistema electoral español, Madrid, 2004; SHUGART, "Comparative electoral...», cit., 2005; SHUGART et al., "Looking for Locals: Voter Information Demands and Personal Vote-Earning Attributes of Legislators under Proportional Representation», American Journal of Political Science, $\mathrm{n}^{\circ}$ 29, 2, 2005, p. 437449). Sin embargo, a pesar de existir profundos y completos análisis, aún falta una investigación general que compare los países que adoptan una representación proporcional de lista preferencial, al menos que yo sepa.
} 
el recurrido estudio comparado de sistemas electorales y sus consecuencias en algunos aspectos del sistema político, como el ENP, variables socioeconómicas, estructuras gubernamentales y parlamentarias, etc., me centraré en el modo en el que los sistemas de voto preferencial influyen en las dinámicas internas de los partidos. De este modo, este proceder ratifica la afirmación de Shugart de que «corregir la ausencia parcial de datos sistemáticos dentro del partido para aquellos sistemas electorales que impliquen una competencia interna dentro del mismo debe ser una prioridad en los próximos años» ${ }^{6}$. Esta investigación nace de una situación donde para la «mayoría de preguntas sobre el voto preferencial, su estudio está justo ahora empezando» ${ }^{7}$. Sin embargo, aunque el estado del estudio sobre el voto preferencial no es tan dantesco como describía acertadamente Kartz hace más de treinta años, la falta de información, de datos y de análisis continúa, como detallaré.

Si solo tenemos en cuenta las opciones del votante, entonces el sistema del voto preferencial puede ser considerado como un sistema en el que a los votantes no sólo se les ofrece «elegir entre partidos, sino que también pueden elegir entre candidatos individuales que representan al partido de su elección» ${ }^{8}$. No obstante, la elección de los votantes es sólo una parte del estudio, la representación proporcional de lista abierta (RPLA, OLPR) a la que se refiere esta última definición, no es otra cosa más que una categoría de los regímenes PRPL, aunque apenas el más común y difuso, y probablemente el más conocido.

Los efectos de los sistemas electorales en los sistemas de partido ha sido un tema recurrente en la doctrina. El impacto de los sistemas electorales en el ENO, el nivel de desproporcionalidad, y la estructura de la papeleta, han sido señalados como «de especial importancia en el caso de los sistemas de lista» ${ }^{9}$. Sin embargo, antes de comenzar con el análisis de los sistemas de voto preferencial será necesario realizar una aclaración conceptual para identificar una definición compartida. Hay grandes diferencias entre los distintos autores sobre este tema, principalmente porque no ha habido un análisis comparado de casos dentro de una misma categoría. Por ejemplo, Farrell señala que «la mayoría de los sistemas de lista operan con una estructura de votación más flexible o 'abierta', y son, en distintos grados, sistemas preferenciales» ${ }^{10}$, y a continuación enumera aquellos menos abiertos. Además, el autor añade que «una categoría intermedia de apertura se encuentra en casos como Finlandia o Italia [...] donde los votos personales tienen una influencia real en el rango legal del candidato» ${ }^{11}$. Siguiendo esta tendencia,

6 SHUGART, «Comparative electoral systems research: The maturation of a field and new challenges ahead», cit., p. 50 .

7 KATZ, «Intraparty preference...», cit., p. 102.

8 Karvonen, «Preferential voting...», cit., p. 203.

9 FARrell, Electoral systems. A comparative introduction, New York, 2001, p. 82.

10 Ibid., p. 82.

11 Ibid., p. 87. 
Lijphart incluye el voto único transferible de Irlanda (STV) junto con otros sistemas de voto preferencial ${ }^{12}$. Considero que la falta de análisis comparado sobre los casos de listas de preferencias en la representación proporcional ha originado la necesidad de un marco teórico común, especialmente en lo concerniente a los efectos de los sistemas electorales, tanto en las dinámicas intrapartidarias de la rotación parlamentaria, como en la competencia interna.

Por lo tanto, tal y como se ha mencionado previamente, una de las áreas más interesantes para investigar en el campo de los sistemas de voto preferencial es su impacto en la competencia interna de los partidos. Además, aunque no sea analizado en este artículo, debemos preguntarnos si los partidos tienen en cuenta las expectativas de los votantes cuando eligen a un candidato de la lista, lo que todavía desconocemos y está siendo explorado ${ }^{13}$. Más allá de las inferencias impresionistas, la única información disponible la constituye el estudio de casos particulares. También debemos cuestionarnos de qué forma el voto preferencial puede afectar a los partidos políticos, si las preferencias importan en términos de personal de partido y en cómo las estrategias de los partidos difieren en los supuestos de voto preferencial. De este modo, tal y como Carey y Shugart afirman en su conocido artículo, «es ampliamente reconocido que las reglas electorales determinan el grado en el que de forma individual los políticos pueden beneficiarse electoralmente desarrollando y trabajando una reputación distinta de la de su partido» ${ }^{14}$.

A pesar de todo, nuestro interés se centra en los efectos del voto preferencial en la elección del votante y el respaldo de candidatos y su sustitución. De hecho, centrarse en el voto preferencial supone «observar más detenidamente un aspecto de la competencia intrapartidaria» ${ }^{15}$, también considerada como un elemento de la estabilidad electoral y del balance interno de poder de los partidos. Tal y como señaló Shugart, «el estudio de la dimensión interna de los partidos ha sido interrumpido en ocasiones por una difusa caracterización de las variables dependientes por falta de los suficientes datos y, lo que es aún peor, por falta de una correcta comprensión de cuáles son las reglas que se investigan en todos los países ${ }^{16}$.

De acuerdo con Carey y Shugart ${ }^{17}$, cuyos estudios se centraron en la reputación personal del candidato, hay cuatro tipos distintos de sistemas PLPR.

12 Shugart, «Comparative electoral systems research: The maturation of a field and new challenges ahead», cit., p. ix.

13 ANDrÉ et al., «Public and politicians' preferences on priorities in political representation: the consequences of an unexplored gap», en DESCHOUwer, Mind the Gap: political participation and representation in Belgium, Colchester, 2017.

14 CARey and Shugart, «Incentives to Cultivate a Personal Vote: A Rank Ordering of Electoral Formulas», Electoral Studies n ${ }^{\circ} 14,4,1995$, p. 418. Véase también Fenno, Home Style: House Members in Their Districts, Longman, 1978; UsLaner, American Foreign Policy Making and the Democratic Dilemmas, New York, 1985.

15 WildGEN 1985, p. 947.

16 Shugart, «Comparative electoral systems research:...», cit., p. 36.

17 Carey and Shugart, «Incentives to Cultivate a Personal Vote:...», cit. 
En primer lugar, la fórmula de lista abierta con voto múltiple en las que «las listas siguen formadas por los líderes de los partidos, pero en las que los votantes pueden expresar su preferencia por algunos candidatos por encima de otros [...] El que los votantes tengan voto múltiple [...] significa que los candidatos de un partido determinado pueden correr como un bloque» ${ }^{18}$ (un ejemplo de esto se puede ver en Italia con anterioridad a las reformas de 1993) ${ }^{19}$.

En segundo lugar, las listas abiertas y de un único voto. En estos sistemas, «los votantes pueden emitir un único voto por debajo del nivel de partido, ya sea para un concreto candidato o una lista de facciones $[\ldots]$ los candidatos no podrán correr como equipos, buscando compartir los votos particulares de unos votantes. Cada candidato o cada lista está sola en el cuestionario de voto único de cada votante ${ }^{20}$ (algunos ejemplos de esto son Brasil, Chile y Polonia).

En tercer lugar, las fórmulas de lista abierta con respaldo abierto y votos múltiples, donde «los líderes de los partidos no tienen la capacidad de seleccionar candidatos $»^{21}$. No hay ejemplos de este sistema por el momento.

Por último, las fórmulas de lista abierta con respaldo abierto y voto único. «El sistema de lista abierta en la que los líderes de los partidos no pueden respaldar a nadie y en los que los votantes emiten un único voto por debajo del nivel de partido ${ }^{22}$ (algunos ejemplos son Finlandia y Brasil ${ }^{23}$ ).

El término «preferencial» ha sido objeto de debate, continuando una cierta confusión y excesos en la construcción de su concepto a la hora de dar una definición. Frecuentemente se produce cierto solapamiento entre términos y a menudo una sola palabra se utiliza para referirse a distintos sistemas. Por lo general, hay más de un sistema en los contextos mayoritarios y proporcionales que permite a los votantes emitir un «voto preferencial», es decir, un voto para su(s) candidato(s) favorito(s). Sin embargo, son pocos los sistemas que dan un papel realmente influyente a la decisión del votante. Se necesita una mayor claridad conceptual y teórica para hacer esta comparación posible y realmente valiosa. Como ejemplo, tanto el voto único no transferible, como el voto único transferible, como los sistemas de listas PLPR, permiten a los votantes emitir votos preferenciales. Sin embargo, lo hacen a través de mecanismos diferentes y con consecuencias distintas para el poder mismo de los votantes y las dinámicas intrapartidarias.

18 Ibid., p. 426.

19 PAssarelli, «Determinants of Preferential Voting in Italy. General Lessons from a Crucial Case», Representation, vol. 53, 2017.

20 Carey and Shugart, «Incentives to Cultivate a Personal Vote:...», cit., p. 427.

21 Ibid.

22 Ibid., p. 428.

23 El Sistema brasileño se aproxima mucho a esta configuración, donde una vez que el político es elegido bajo una etiqueta de partido no se le puede negar el acceso a la lista de partidos en elecciones posteriores (Mainwaring, «Politicians, Parties, and Electoral Systems: Brazil in Comparative Perspective», en Comparative Politics, $\mathrm{n}^{\circ} 24$ (1), 1991). 
Concretamente, los votos de los candidatos en los sistemas PLPR se agrupan por partido, mientras que en los otros no es asi ${ }^{24}$.

En general, se puede afirmar que la representación proporcional con voto preferencial - PLPR - concede a los votantes la oportunidad de elegir tanto al partido como a sus representantes parlamentarios, pero el simple hecho de tener la oportunidad de indicar sus preferencias no significa que realmente importen para determinar los resultados electorales. Esto es relevante primero porque - como comentaré más adelante- hay sistemas de lista flexible en los que los votantes sólo pueden aprovechar esta oportunidad si se cumplen ciertas condiciones previas. En segundo lugar, porque incluso en los sistemas de lista abierta reales, cuando el comportamiento del votante decide directamente quien es elegido, la elección de la élite del partido en la lista de candidatos disponibles (más popular, etc.) puede afectar las opciones realistas de otros competidores, produciendo un efecto dominó que acabe limitando las opciones de los votantes antes de que incluso lleguen a las urnas.

Además, como aludiremos más tarde con mayor detalle, el poder del votante en los sistemas PLPR varía considerablemente entre los distintos casos y sistemas dependiendo del número de votos que puedan ser emitidos, la existencia de umbrales, la votación y la obligatoriedad.

La principal característica de los sistemas de voto preferencial es su capacidad - a pesar de lo que ocurre en otros sistemas electorales- de aumentar la influencia de los votantes en la elección de los representantes. Igualmente, de acuerdo con un importante sector de la doctrina y de la política, se reclama que el voto preferencial debería aumentar la responsabilidad de los parlamentarios. Sin embargo, esta suposición debe ser aún contrastada —al menos que yo conozca- $y$, en cualquier caso, no de forma comparada. Parece que, en este sentido, el voto preferencial se entiende como un sistema en el que los votantes eligen directamente a los parlamentarios, aunque sin implicar que estos determinen quien es elegido. En esta corriente de pensamiento, es relevante mencionar la distinción que realiza Pedersen (1966) dependiendo de si el voto preferencial se utiliza o no de manera efectiva como un modo de comprobar la tesis de la responsabilidad. Nuevamente, la verdadera capacidad de los votantes para influir en la elección de parlamentarios y, tal vez, en sus responsabilidades, es la de medir el poder de los votantes en el candidato que eligen. Me comprometeré a tratar estas medidas de un modo empírico.

Matthew Shugart, en su valiosa aportación al libro editado por Gallagher y Mitchell sobre el estado de la investigación de los sistemas electorales, señala claramente que «quizás sirva para promover el desarrollo de la investigación en la gran categoría de los sistemas PR de lista preferencial (PLPR), si los orientamos

24 Afecta a la distribución de escaños entre partidos y candidatos cuando los votos agrupados también cuentan para el partido. 
claramente hacia otros sistemas que puedan ser definidos como 'preferenciales' ${ }^{25}$, por lo que al adoptar esta propuesta el mencionado libro ofrece un análisis e información del voto preferencial (PV) desde una perspectiva comparada, incluyendo a más de 20 países de todo el mundo. El voto preferencial es un tipo concreto de voto a disposición de los votantes en los sistemas PR. Esta investigación se centra en los sistemas de listas abiertas y flexibles, aquellos en los que los votantes pueden (y en algunos casos deben) emitir una preferencia (y en algunos casos más de una) de los candidatos disponibles en la lista del partido. De este modo, el libro aborda los efectos de los sistemas electorales, concretamente los de aquellos sistemas que han adoptado un modelo de voto preferencial. El objeto de la investigación es de especial interés en política comparada, no sólo para aquellos que estudien sistemas electorales, sino también aquellos interesados en las consecuencias relacionadas con las características del voto preferencial, así como en sus efectos en los partidos, en los votantes y en los candidatos. Esto representa una perspectiva nueva e innovadora desde la que estudiar el voto preferencial. De hecho, salvo algunas notables excepciones relativamente recientes ${ }^{26}$, el voto preferencial ha sido muchas veces olvidado en los estudios comparados de sistemas electorales, especialmente en términos de análisis de datos. A pesar de la importante doctrina existente en sistemas electorales, el voto preferencial se incluye normalmente en el análisis general de los diferentes sistemas electorales de cada país ${ }^{27}$. Lo que es más, la caracterización de los sistemas de voto preferencial es claramente escasa y carece del suficiente análisis comparativo. No obstante, en los últimos diez años un número mayor de autores han dirigido sus estudios al voto preferencial $^{28}$. Además, han aparecido nuevos estudios centrados en algunos aspectos relacionados con el voto preferencial (género, votos personales, características personales de los candidatos, etc. ${ }^{29}$. A pesar de todo, aunque existan análisis válidos y estudios pormenorizados de casos concretos, todavía es necesario una investigación global que compare países que adopten o una «representación proporcional de lista abierta»(OLPR) o sistemas de mayor flexibilidad. Si es

25 SHUGART, «Comparative electoral systems research: ...», cit., p. 40.

26 KATZ, A Theory of Parties and Electoral Systems, Johns Hopkins University Press, Baltimore, 1980; del mismo, «Le nuove leggi per l'elezione del Parlamento», en Politica in Italia, 1994, pp. 161-186; MARSH, «The Voters Decide?», cit., 1985.

27 Gallagher and Mitchell (eds.), The Politics of Electoral Systems, Oxford, 2005; Colomer, 2010.

28 Shugart, «Comparative electoral systems research:...», cit.; Karvonen, The Personalization of Politics: a study of parliamentary democracies, Colchester, 2010; del mismo, «Preferential Vote in Party List», cit.; Farrell, Electoral Systems. A comparative introduction, MacMillan, 2011.

29 Bergman et al, 2013; KARVONEN, «Preferential Voting: Incidence and Effects», cit.; del mismo, «Preferential Vote in Party List», cit.; KATZ, «Intraparty Preference Voting», cit.; MARSH, «The Voters Decide?», cit.; Ortega, Los sistemas de voto preferencial..., cit.; PASSARElli, «Determinants of Preferential Voting in Italy», cit.; SHUGART, «Comparative Electoral Systems Research», cit.; SHUGART et al., «Looking for Locals: Voter Information Demands and Personal Vote-Earning Attributes...», cit.; VALDINI, «A deterrent to diversity: The conditional effect of electoral rules on the nomination of women candidates», en Electoral Studies, $\mathrm{n}^{\circ}$ 31(4), 2006, p. 740-49; WAUTERS et al. 2016. 
cierto, tal y como Katz y Bardi afirman — haciéndose eco de lo expresado por Shugart-, que «el voto preferencial ha recibido menos atención por los autores de la que debería ${ }^{30}$, este trabajo comparando una amplia selección de casos de relevancia aspira modestamente a colmar esa laguna, la cual abarca al menos tres amplios campos de investigación. Esta ausencia a nivel cuantitativo se caracteriza por la falta de una base de datos suficiente sobre el voto preferencial que cubra un gran lapso de tiempo y que ahonde más allá de los datos a nivel nacional. A nivel cualitativo supone la escasez de datos de partidos y del personal político. Por último, hay una ausencia de una investigación detallada que examine las consecuencias del uso del voto preferencial.

Además de todo ello, han aparecido numerosas cuestiones dignas de estudio sobre la «bondad» del voto preferencial, así como de su efecto en las «cualidades» del personal de los partidos, en la organización del partido, en el poder de los votantes, en los candidatos y en la selección de parlamentarios (MP). Partiendo de una descripción de los diferentes tipos de sistemas electorales (nacional y europeo) que posibilite a los votantes votar de forma preferencial, comprobaré la hipótesis de que el voto preferencial tenga impacto en las dinámicas intrapartidarias y en el sistema político y de partidos. En concreto, se presentarán diferentes variables en aras de medir las consecuencias del voto preferencial en la competencia dentro del partido y la estructura del sistema político y de partidos. Mediante la recopilación de datos trasnacionales, este trabajo pretende colmar las mencionadas carencias en el progreso cuantitativo, cualitativo y analítico. Cabe señalar que, debido a las restricciones para la recopilación de datos, la medida en la que estas encuentran solución variará en cada país. La presente investigación expande el entendimiento del voto preferencial de dos modos. Por un lado, proporciona un conjunto de datos sistemáticos sobre el voto preferencial. Por otro, pone a prueba la hipótesis sobre los efectos del voto preferencial.

Entre la doctrina, existen numerosas definiciones de los sistemas electorales que permiten al votante expresar su voto preferencial, centrándose en diferentes aspectos del mismo. Algunas focalizan su atención en el poder conferido al votante, tales como cuántos votos preferenciales puede emitir el votante; es importante que tales definiciones tomen en cuenta si el votante puede cambiar o no el orden de la lista de cada partido o votar por el candidato de otro partido distinto. Nuevamente, continúa habiendo preguntas centrales ¿son los resultados electorales decididos por las preferencias de los partidos, por las de los votantes o por las de ambos? ¿Es el voto preferencial obligatorio u opcional? ¿Hay algún requisito, como un voto basado en género, en el caso de que se permita más de una preferencia?

30 KATZ y BARDI, «Voto di preferenza e ricambio del personale parlamentare», en Rivista Italiana di Scienza Politica, 1979, vol. IX, n. 1, p. 94. 
¿Existe un «perfecto» ${ }^{31}$ «conjunto de normas que estructure cómo son emitidos los votos para las elecciones de una asamblea representativa y como estos se convierten en escaños» ? $^{32}$ Si semejante sistema existiese, ¿sería deseable tenerlo? No cabe duda de que la última pregunta es retórica, pero no por ello menos importante. Muchos politólogos han intentado dar con el mejor sistema electoral ¿Se ha salvado de alguna manera la gran división entre los sistemas mayoritarios y proporcionales para crear «lo mejor de los dos mundos», como se pidió retóricamente en un libro sobre sistemas electorales mixtos? ${ }^{33}$. La efectividad y «calidad» de un sistema electoral viene determinada en gran medida por el sentimiento de los actores políticos hacia él y la medida en la que se ajusta a sus objetivos y expectativas. De este modo, la atribución de «perfección» a un sistema dependerá por lo general de los fines para los que fue adoptado o para los que se ha propuesto su uso futuro. El ciudadano de una antigua ciudad-Estado griega (polis) probablemente desearía un sistema de representación proporcional que incluyese a todos los ciudadanos. En cambio, la élite gobernante seguramente preferiría un sistema que minimizase la influencia de los poderes políticos que están compitiendo ${ }^{34}$. Los sistemas electorales de representación proporcional son en general favorecidos por los pequeños partidos y por las minorías (lengua, etnia, religión, territorio, etc.). Las minorías aceptarán un sistema mayoritario (distrito de un solo escaño - SSD) cuando se presuma que pueden elegir algunos candidatos por concentración geográfica de sus votos en un área concreta ${ }^{35}$. Por el contrario, los grandes y medianos partidos, más repartidos geográficamente en sus bases nacionales, son capaces de maximizar sus resultados electorales tanto en sistemas de representación proporcional como en los mayoritarios, como los de SSD o los de doble votación. De este modo, evaluar la «perfección» de un sistema electoral no es tarea fácil y casi por definición conlleva cierto grado de subjetivismo. Para un determinado sistema electoral, el análisis y evaluación de sus características y funcionamiento debe considerar los efectos en distintas áreas y en diferentes actores sociales y políticos, por lo que cualquier conclusión sobre su efectividad estará siempre sesgada por el parámetro que el evaluador haya elegido.

En primer lugar, es necesario describir brevemente las principales características de los sistemas más comúnmente adoptados. Por lo general, los sistemas electorales se dividen en mayoritarios y proporcionales. La mayoría de los estudios y debates han rondado las características y efectos de los sistemas plurales

31 ¿Para quién y para qué? Hay muchas variables a considerar y, a veces, difieren en los resultados, aunque no siempre son realmente comparables. Los partidos, los votantes, los candidatos, la responsabilidad, la rotación, la participación son sólo unos pocos aspectos de los sistemas electorales.

32 Gallagher y Mitchell, The Politics of Electoral Systems, cit., p. 3.

33 Shugart y Wattenberg, Mixed-Member Electoral Systems. The Best of Both Worlds?, Oxford, 2003.

34 Manin, The Principles of Representative Government, Cambridge, 1996.

35 Sartori, Parties and party systems, 1976; Taagepera y Shugart, Seats and Votes. The Effects and Determinants of Electoral Systems, New Haven \& London, 1989. 
frente a los de los proporcionales, distinción marcada por el factor geográfico por el cual las naciones del Oeste de Europa tradicionalmente han adoptado un sistema de representación proporcional, mientras que los países angloamericanos están asociados con los mayoritarios, el sistema de «primer paso» o sistema SSD. Dentro de las más amplias categorías que Michael Gallagher sintetiza, podemos identificar tipos más precisos de sistemas electorales, como: 1) pluralidad de un solo miembro (SMP), voto alternativo (AV), sistemas de dos rondas (2RS) para la categoría de circunscripción de un solo miembro; 2) mitad compensatorio mitad paralelo para los sistemas mixtos; 3) sistemas de lista cerrada; 4) Lista abierta y lista flexible para la categoría de sistemas de lista preferencial (a los que debemos añadir al menos otros dos subtipos, que veremos más tarde); y el voto único transferible y proporcional, y el SNTV (adoptado en Japón) ${ }^{36}$.

La doctrina más consolidada sobre sistemas electorales acepta que varios factores principales deben ser considerados en cualquier análisis comparado. Desde un punto de vista meramente teórico, los principales factores a tener en cuenta en los sistemas electorales son: el tipo de voto (obligatorio o no), la estructura de la votación, el número de votos permitidos, la posibilidad de separarlo, la influencia del votante en la elección de los candidatos, la magnitud del distrito, el número de circunscripciones, la existencia de un umbral legal, el voto preferencial, la previsión de escaños de compensación, el número de niveles. De acuerdo con $\mathrm{Rae}^{37}$, una de las características más importantes de su sistema electoral es la fórmula que adopte, así como la presencia de un umbral legal y la naturaleza de la elección en la que los votantes se expresan (lista de partido, candidato o ambas). Es cierto que los sistemas electorales pueden influir en los votantes, en los candidatos, en los partidos, en las políticas y en el sistema de partidos. Además de todo ello, algunos sistemas electorales permiten al votante emitir más de un voto, que se puede lograr al permitir a los votantes elegir tanto a un candidato como a una lista (el caso de Alemania, por ejemplo), o emitir votos para más de una preferencia distinta (el caso de Italia con anterioridad a 1991, el cual, por cierto, no es mencionado por Gallagher y Mitchell, puesto que seguramente consideren el uso de más de una preferencia como un voto/ un caso per se $)^{38}$. En esta corriente de pensamiento es importante también mencionar la estructura de la votación como un elemento crucial de los sistemas electorales. En realidad, distinguimos entre sistemas donde sólo se permite a los votantes votar por un partido o sistemas en los que los votantes pueden clasificar partidos y/o candidatos.

Como se ha dicho antes, hay campos en los que la doctrina está consolidada, como aquellos concernientes a los efectos de los sistemas electorales en el número

36 Gallagher y Mitchell, The Politics of Electoral Systems, cit.; Shugart y Wattenberg, Mixed-Member Electoral Systems. The Best of Both Worlds?, Oxford, 2001, p. 11.

37 RAE, The political consequences of electoral laws, New Haven, 1967.

38 Gallagher y Mitchell, The Politics of Electoral Systems, cit., p. 7. 
de partidos o el nivel de desproporcionalidad ${ }^{39}$. Sin embargo, hay otros muchos aspectos relevantes de los sistemas electorales que continúan fragmentados o que requieren de un mayor análisis por la doctrina, como el impacto que los sistemas pueden tener en la composición interna de los partidos políticos. En este trabajo, me fijaré en el efecto que un particular sistema electoral tiene en los partidos y en la representación, así como otras variables que pueden influir positivamente en el comportamiento del votante a la hora de decidir una determinada preferencia. En los sistemas mayoritarios, tanto en los plurales como en los mayoritarios, la elección del votante puede ser realmente decisiva, debido a la posibilidad de elegir directamente entre los candidatos del distrito, por lo que el votante puede acabar influyendo en el resultado final sin limitarse a una lista de partido. Dicha influencia variará dependiendo de la «apertura» de la segunda votación en sistemas mayoritarios (2RS), pero suele ser de cierta relevancia. En cambio, en los sistemas proporcionales, los votantes votan por un partido y el reparto de escaños entre los candidatos es decidido siguiendo el orden de la lista de partido. No obstante, resulta «un error referirse a 'representación proporcional', como si esta fuese un único tipo de sistema electoral cuando realmente es un término genérico que abarca un gran número de diferentes sistemas, los cuales únicamente comparten el fin común de proporcionalidad entre los votos y los escaños $»^{40}$. Además, también hay sistemas con «listas abiertas»: aquellos en los que los votantes pueden indicar su preferencia por un candidato ${ }^{41}$. Hay igualmente sistemas electorales de miembros mixtos que combinan aspectos de representación proporcional y sistemas plurales. Esta combinación se alcanza introduciendo elementos compensatorios mixtos (con escaños adicionales) o elementos paralelos mixtos en los procedimientos por los que se asignan escaños. En los últimos tiempos, estos sistemas mixtos han experimentado una gran difusión mundial y el número de países que lo han adoptado ha aumentado rápidamente hasta un total de doce, aunque lejos queda la gran ola de difusión en los noventa ${ }^{42}$.

Existe una doctrina consolidada en el estudio de los sistemas electorales que ha establecido dos objetivos sobre diversos aspectos de su investigación. De hecho, podemos distinguir entre dos grandes enfoques, uno centrado en la clasificación de los sistemas electorales, y otro - construido a partir del primerocentrado en clasificar los efectos de estos sistemas. Será especialmente importante prestar atención al proyecto de clasificarlos, por la necesidad de que el voto preferencial debe ser clasificado de forma singular, así como a la definición de qué entendemos por voto preferencial, dónde debe ser colocado en la clasificación de sistemas electorales, cuáles son sus principales características y qué problemas

39 TaAgepera y Shugart, Seats and Votes. The Effects and Determinants of Electoral Systems, cit., p. 18.

40 Bogdanor, Multi-party Politics and the Constitution, Cambridge, 1983, p. 2.

41 Marsh, «The Voters Decide?», cit.; Katz, «Intraparty Preference Voting», cit.

42 Massicotte 2011; Shugart and Wattenberg, Mixed-Member Electoral Systems: The Best of Both Worlds?, Oxford, 2001. 
dificultan su estudio. Por lo tanto, el estudio de cómo clasificar los sistemas electorales será vital para explicar posteriormente los efectos del voto preferencial. Si consideramos que las elecciones están básicamente representadas por sus fórmulas electorales, es decir, la regla que determina cómo se traducen los votos en escaños, es realmente importante centrarse en el voto preferencial para proporcionar más información e interpretaciones

No obstante, la doctrina ha recurrido a diferentes enfoques y métodos para hacer una clasificación de los sistemas electorales, teniendo cada uno de ellos sus propios indicadores, variables y criterios analíticos, y surgen de diversos marcos teóricos que finalmente generan muchas más clasificaciones, grupos y consecuencias analíticas.

Por lo general, las clasificaciones de sistemas electorales han seguido el criterio de diferenciar entre pluralidad, sistemas mayoritarios y representación proporcional, a las cuales debe sumarse ahora la categoría mixta ${ }^{43}$. Bajo dichas categorías, encontramos sistemas electorales que generalmente siguen el mismo razonamiento y que apenas varían entre uno y otro. Se pueden identificar dos distintos enfoques entre los autores: el primero alude a la fórmula electoral y sigue el estudio seminal de Arend Lijphart ${ }^{44}$, proponiendo una lista detallada de los distintos sistemas y sus funcionalidades. El segundo enfoque considera de gran importancia otros elementos distintos de la fórmula electoral a la hora de clasificar los sistemas electorales. Distintos autores han subrayado la importancia de muchos factores para una apropiada clasificación de los sistemas. Douglas Rae ha hecho hincapié en la diferencia entre dos tipos de papeleta ${ }^{45}$ : las papeletas categóricas que «obligan al votante a elegir un candidato o partido»; las papeletas ordinarias que «permiten al votante expresar una preferencia más compleja y equívoca al ordenar los partidos» o los candidatos ${ }^{46}$. Además, Rae ha introducido también el concepto de sistemas electorales centrados en el candidato o centrados en el partido ${ }^{47}$, mientras que Taagepera y Shugart subraya el «papel decisivo» de la magnitud del distrito para influir en la proporcionalidad ${ }^{48}$. André Blais ha intentado ir más allá de la distinción entre pluralidad, mayoría y proporcionalidad al diferenciar tres dimensiones de los sistemas electorales: la papeleta, la estructura y la fórmula, dividiéndolas, a su vez, en seis categorías: «(1) La naturaleza de la circunscripción (completa o por distritos); (2) magnitud de la circunscripción; (3) el objeto de la votación (listo o individuo); (4) el número de votos permitidos;

43 Shugart and Wattenberg, Mixed-Member Electoral Systems: The Best of Both Worlds, cit.

44 Lijphart, Electoral systems and party systems. A Study of Twenty-Seven Democracies, 1945-1990, Oxford, 1994.

45 Gallagher y Mitchell, The Politics of Electoral Systems, cit., p. 7.

46 RAE, The political consequences of electoral laws, cit., pp. 17-19; 1971 (edición revisada).

47 Shugart, «Electoral «Efficiency» and the Move to Mixed-Member Systems», en Electoral Studies, $\mathrm{n}^{\mathrm{o}} 20,2001,173-193$.

48 TaAgepera y Shugart, Seats and Votes. The Effects and Determinants of Electoral Systems, cit., p. 112. 
(5) el tipo de voto (nominal/ordinario/numérico); y (6) la fórmula (mayoritaria/ pluralidad/proporcionalidad)» ${ }^{49}$. Por último, David Farrell clasifica los sistemas electorales de acuerdo con la fórmula electoral y su resultado, y ofrece una tipología basada en la estructura de la papeleta (elección basada en candidatos o en partidos), y el alcance de la elección del votante (categórica/ordinal). Gallagher y Mitchell ${ }^{50}$ plasman en una lista detallada las principales dimensiones de los sistemas electorales, especialmente la magnitud del distrito, la estructura de la votación y el número de votos emitidos, así como un estudio de distintos casos y ejemplos.

La elección de un sistema electoral es importante en muchos aspectos. Puede afectar al sistema político, al sistema de partidos, a la naturaleza del gobierno, a la cohesión de los partidos, a la influencia de los votantes en la elección de candidatos, al nivel de responsabilidad, etc. Los sistemas electorales influyen en las acciones de los actores políticos. Hay varios elementos de los sistemas electorales que pueden afectar a los partidos políticos en su magnitud, organización y actividades de campaña, así como las oportunidades para candidatos independientes en un sistema político. Fundamentalmente, el comportamiento de los partidos políticos representa su respuesta a las posibilidades y limitaciones de un sistema dado. Los sistemas electorales tienen efectos mecánicos y psicológicos (Duverger 1951). Mecánicamente, su consecuencia es observable en la transformación de votos en escaños. Psicológicamente, afectan tanto a los votantes como a los partidos de diferentes maneras. Antes de la votación, los votantes deciden estratégicamente qué partido o candidato apoyar. Esta elección se basa, en parte, en una consideración de que su voto cuente ${ }^{51}$. Sin embargo, tales consideraciones estratégicas operan de manera diferente en los sistemas electorales. Los votantes responderán a las oportunidades y limitaciones del sistema, lo que significa que tomarán diferentes decisiones en un sistema de pluralidad que en un sistema OLPR. Del mismo modo, los partidos políticos se ven afectados por los sistemas electorales y reaccionan en consecuencia. Los líderes del partido deciden, por ejemplo, nominar a un político (o no) en un distrito determinado, hacer alianzas con otros partidos o postularse solos. Si consideramos las dos categorías principales de sistemas electorales (mayoritarios versus proporcional), más las mixtas, se pueden observar diferentes efectos dependiendo de las características de un sistema electoral dado. Estos elementos determinan diferentes resultados en los campos de partidos/candidatos y votantes.

En el caso de la magnitud de la circunscripción, surge rápidamente una gran distinción categórica. Bajo un sistema mayoritario (FPTP, SSD, AV o el sistema de dos rondas), los votantes eligen a un solo representante. Por el contrario, todos

49 Ibid.

50 Gallagher y Mitchell, The Politics of Electoral Systems, cit., pp. 5-9.

51 Cox, Making votes count, cit. 
los sistemas proporcionales ${ }^{52}$ requieren circunscripciones electorales que elijan a más de un miembro; de lo contrario, su lógica imitará la del SSD ${ }^{53}$. Bajo cualquier sistema proporcional, el número de miembros a ser seleccionados en cada circunscripción determina, en gran medida, cuán proporcionales serán los resultados de las elecciones. Además, generalmente existe una fuerte correlación positiva entre la magnitud promedio de la circunscripción y la magnitud promedio del partido. En consecuencia, la magnitud del partido es un factor crucial para determinar quién será nominado para la candidatura y, finalmente, elegido. Es probable que cada sistema electoral fomente diferentes tipos de organización de partidos. Por ejemplo, es probable que los sistemas políticos proporcionales con listas cerradas favorezcan a las partidos políticos fuertes. Por el contrario, los sistemas descentralizados basados en distritos como FPTP pueden tener el efecto contrario. Del mismo modo, PLPR puede favorecer, o no desincentivar, como mínimo, la competencia dentro de los partidos y los conflictos o divisiones.

El tipo de sistema electoral también puede influir en el tipo de sistema de partidos que se desarrolla. Maurice Duverger (1951) se refirió a la influencia del sistema electoral en el sistema de partidos al presentar las conocidas y debatidas tres leyes sociológicas: (1) un voto mayoritario en unas elecciones es propicio para un sistema bipartidista; (2) la representación proporcional es favorable para un sistema multipartidista; (3) un voto mayoritario en dos elecciones propicia un sistema multipartidista, inclinado a formar coaliciones. Aunque no existe un

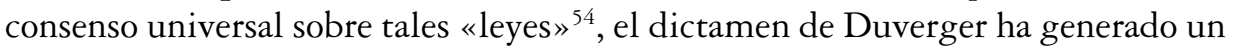
debate generalizado. En particular, el efecto de los sistemas «mayoritarios» en la reducción tanto del número de partidos como de los candidatos «reales» ha sido comprobado de diferentes formas ${ }^{55}$. Del mismo modo, otro aspecto que, según

52 Además, este es también el caso de algunos sistemas mayoritarios como los de votación conjunta y PBV, y algunos otros sistemas como los de voto limitado y SNTV. Entre los casos históricos también mencionaría el Senado italiano (1948-1993), que tenía una fórmula dual. Funcionaría como un sistema SSD donde un candidato obtuviera el $65 \%$ de los votos; los escaños no completados en SSD fueron asignados en su lugar a nivel regional a través del sistema D'Hondt (PASSARELli, «Electoral system in context: Italy», en The Oxford Handbook of Electoral Systems, Oxford, 2018, p. 867), o el sistema utilizado desde las elecciones de 2012 en Rumania, donde solo aquellos candidatos que obtienen más del $50 \%$ de los votos en circunscripciones uninominales son elegidos automáticamente. Los escaños restantes se distribuyen entre los partidos políticos primero a nivel de condado (utilizando la cuota Hare) y luego a nivel nacional (según el sistema D’Hondt), siempre que superen el umbral del $5 \%$.

53 Shugart, 2018.

54 SHUGART, «Comparative electoral systems research: ...», cit., p.30.

55 Benoit, 2002; Cox, Making votes count, cit.; TaAgepera y Shugart, Seats and Votes..., cit.; LijPHART, Electoral systems and party systems ..., cit.; GrofmAn, 1985.

Recientemente, Shugart y TAagepera, Votes from seats: ..., cit., han introducido innovadoras herramientas teóricas y empíricas para deducir el número de partidos cuando solo se conoce el número de escaños en una asamblea representativa. TAagepera. Predecir el tamaño de los partidos. La lógica de los sistemas electorales simples, Oxford, 2007, se centró en las revisiones a introducir en los sistemas electorales para obtener el cambio deseado en el número promedio de partidos y la duración del gobierno. 
Shugart, merece más atención es el papel de los sistemas de dos niveles en la asignación de escaños ${ }^{56}$.

Además, los sistemas electorales afectan al tipo de relaciones que se forman entre los candidatos individuales y sus partidarios. Los sistemas que hacen uso de distritos electorales uninominales pueden alentar a los candidatos individuales a verse a sí mismos como delegados de áreas geográficas particulares, y comprometidos con los intereses de su electorado local. Por el contrario, los sistemas que utilizan grandes circunscripciones plurinominales, como la mayoría de los sistemas proporcionales, son más propensos a aportar representantes cuya lealtad principal recae en su partido y en torno a cuestiones nacionales. Si aceptamos que los sistemas electorales influyen en el comportamiento de los partidos, los votantes y los candidatos, entonces debemos considerar las principales dimensiones y problemas de un sistema electoral, y cómo esos elementos distintivos pueden dar lugar a patrones específicos de comportamiento. Entre esos elementos, los más relevantes son: la magnitud del distrito $(\mathrm{M})$, el nivel de los umbrales legales, el tamaño de la asamblea, el nivel de dispersión electoral en el territorio nacional, los escaños adicionales, la doble votación, la fórmula electoral o la papeleta de votación ${ }^{57}$.

En particular, a medida que se realiza la transición a un análisis sobre casos de votación preferencial, se debe enfatizar que las listas abiertas, el voto preferencial, la representación proporcional personalizada y la votación de «lemas» (marcas) y «sublemas» permiten que el votante seleccione un candidato del partido y uno o más candidatos individuales. Las variaciones incluyen preferencias obligatorias u opcionales, esta última permitiendo a los votantes emitir votos para los candidatos individuales o la lista de partidos según lo dado (a menos que, obviamente, se abstengan o el voto sea en blanco o considerado nulo). El procedimiento básico requiere que todos los votos preferenciales para candidatos individuales o listas de partidos se cuenten como votos para el partido correspondiente; después de que a cada partido se le haya asignado un número de escaños relacionados con esos votos, se llenan parcialmente con los candidatos individuales que figuran en las listas. Por supuesto, cuanto mayor sea el número de preferencias individuales que cada votante pueda manifestar en la papeleta, más intensa será la competencia de los candidatos individuales para completar los escaños correspondientes. Los sistemas de lista abierta que permiten al votante seleccionar unos pocos candidatos, generalmente de uno a tres, han sido utilizados en la mayoría de los países de Europa occidental ${ }^{58}$.

56 SHUGART, «Comparative electoral systems research: ...», cit.

57 Cox, Making votes count, cit.; Sartori, Parties and party systems, cit.; TaAgepera y Shugart, Seats and Votes..., cit.; Grofman - LijPhART; RAE, The political consequences of electoral laws, cit.; FARRELL, Electoral systems: A comparative introduction, 2011; GALLAGHER y MiTCHELL, The Politics of Electoral Systems, cit.

58 En Italia desde 1946-1948 hasta 1993. Hasta 4 preferencias hasta 1991 (PASSARELLI, «Determinants of Preferential Voting in Italy», cit.; «Electoral system in context: Italy», cit.). (COLOMER, Handbook of electoral system choice, Palgrave, 2004, p. 51-52). 
El uso de PLPR a menudo va acompañado de un debate sobre las supuestas ventajas y desventajas que trae para los votantes y los partidos. Si bien el análisis de los pros y los contras está profundamente relacionado con la cultura política particular de cada nación, la voluntad de los partidos y los objetivos de los actores políticos, es posible construir un marco de ventajas y desventajas teóricas que generalmente están asociadas, en la mayoría de los contextos, con el PLPR. Muchos académicos culpan al PLPR por debilitar a los partidos y por un comportamiento electoral particular ${ }^{59}$, mientras que otros argumentan que el intercambio oculto detrás del PLPR puede ser «positivo» ${ }^{60}$. En particular, puede haber relaciones positivas entre algunas variables sociopolíticas y el uso del voto preferencial. Según los defensores del PLPR, la introducción de sistemas de votación preferenciales generalmente trae varias ventajas. Permite a los ciudadanos elegir entre diferentes candidatos en las listas, y aumenta la legitimidad y la responsabilidad de los cargos electos. Los votos preferenciales generarían un proceso de rendición de cuentas ya que aumentaría el nivel de democracia dentro del partido, lo que conduciría a una mayor influencia de las ramas locales del partido en la sede central. Aumenta la calidad de los candidatos propuestos y, en consecuencia, sus campañas e introduce un sistema de méritos para crear listas de candidatos y disminuye la probabilidad de proponer candidatos débiles y miembros obedientes del partido. Sin embargo, pueden surgir desventajas cuando examinamos la satisfacción de los votantes ${ }^{61}$. En particular, algunos argumentan que el PLPR conduce a la corrupción y la competencia electoral dentro del partido, lo que puede causar fragmentación y desunión dentro de los mismos. Estos autores encontraron que «el voto preferencial (como se definió anteriormente, y no exclusivamente en los sistemas PLPR - nota del autor) puede marcar una diferencia en los niveles de satisfacción de los votantes con la democracia» ${ }^{62}$. La desunión de los partidos es, de hecho, uno de los principales problemas atribuidos a los efectos del PLPR. La proliferación de facciones, o estancamientos políticos, son algunas de las consecuencias del tipo de competencia causada dentro del partido, o al menos no impedida, por los sistemas PLPR. La unidad del partido se ve socavada por la dura lucha entre los candidatos del mismo partido. El aumento asociado de los costos de la campaña electoral se considera uno de los efectos negativos del PLPR, especialmente debido a su relación positiva con los fenómenos de corrupción en los intercambios de votos y los vínculos negativos de patrocinio entre los

59 Mainwaring, 1991; Ames, 1995; Chang y Golden, «Electoral Systems, District Magnitude and Corruption», en British Journal of Political Science, $\mathrm{n}^{\circ} 37$ (1), 2007.

60 Piattoni, Clientelism, interests, and democratic representation: the European experience in historical and comparative perspective, Cambridge, 2000.

61 Farrell - Mcallister, «Voter Satisfaction and Electoral Systems: Does Preferential Voting in Candidate-Centred Systems Make a Difference?», European Journal of Political Research, $\mathrm{n}^{\circ}$ 45, 2006, p. 723-749.

62 Íbid. p. 743. 
candidatos y los votantes ${ }^{63}$. La competencia interna es una de las características más destacadas de PLPR. Su mayor enfoque en el voto personal puede impulsar a los candidatos con intereses locales sobre aquellos con intereses nacionales más programáticos ${ }^{64}$. Esto puede considerarse una ventaja, en términos de la estrecha relación fomentada entre los candidatos y los votantes y la defensa de los intereses locales, o una desventaja, dado el aumento asociado a la presión ejercida por los lobbies a favor de intereses particulares y los riesgos bien conocidos de patrocinio y corrupción.

Si se puede decir que los sistemas proporcionales presentan una mayor variación que sus homólogos de distritos uninominales, también es evidente, como señaló Eckstein, que «es la cosa más fácil del mundo enredarse entre las complejidades de los sistemas electorales» ${ }^{65}$. De hecho, tal declaración solo se puede aplicar razonablemente al «mundo» de los sistemas de votación preferencial. Aquí, aclararé mejor el uso de lemas, así como los subtipos de este sistema, al presentar una discusión sobre sus efectos en los candidatos, votantes y partidos. Primero, se debe hacer una distinción primaria en la familia de los sistemas proporcionales de listas. Encontramos sistemas de listas en los que los votantes no pueden expresar una preferencia por el candidato en la lista (lista cerrada), y sistemas de listas en los que los votantes pueden emitir una preferencia que afecta la lista (lista preferencial). Para clasificar mejor los sistemas electorales, los académicos han indicado una variedad de factores distintivos importantes como: los tipos de papeletas ${ }^{66}$, el número de votos permitidos, la magnitud de la circunscripción, la presencia de umbrales legales, la segunda vuelta, etc. ${ }^{67}$.

De los casi sesenta países democráticos agrupados por Lawrence LeDuc y Lijphart $^{68}$, en 1999 casi la mitad tenía sistemas de representación proporcional ${ }^{69}$. Entre esos países (y agregando algunos otros no incluidos en esas listas iniciales), el número total de casos de representación proporcional que adoptaron un sistema de votación preferencial fue de 22 en 2017. Por lo tanto, se puede decir que aproximadamente una cuarta parte de los países democráticos del mundo han adoptado sistemas electorales de preferencia, aunque con una variación considerable entre ellos, como el alcance de los poderes de los votantes, el grado de «apertura», el papel de los partidos, etc. (ver infra). Centrándome en PLPR, tengo una muestra considerable de países, con una variación considerable en términos de diseño institucional, ley electoral, tamaño del país, etc. Un simple vistazo a la distribución geográfica ilustra claramente que la mayoría de los 22

63 AMES, 1995.

64 Carey - Shugart, «Incentives to Cultivate a Personal Vote», cit.; Bergman et al. 2013.

65 ECKSTEIN, Comparing politics: a reader, New York, 1963, p. 249.

66 RAE, The political consequences of electoral laws, cit.

67 Gallagher y Mitchell, The Politics of Electoral Systems, cit., p. 7.

68 L. Leduc, 1996; Lijphart, 1999.

69 FARRELL, Electoral systems. A comparative introduction, cit., pp. 8-9. 
países con sistema proporcionales que han tenido (o tuvieron, como en el caso italiano), un sistema de votación preferencial se encuentra en Europa, con solo unos pocos en América Latina, y en los otros continentes tienen escasa representación (o incluimos los sistemas mayoritarios). Esto confirma la observación de Marsh de hace más de treinta años de que en la mayoría de las democracias de Europa occidental (véase el siguiente párrafo), los votantes tenían permitido elegir entre los candidatos de un partido a nivel de elecciones parlamentarias ${ }^{70}$. La votación preferencial también se usa en las elecciones parlamentarias de varios países poscomunistas que adoptaron PLPR para sus regímenes democráticos, mientras que las democracias latinoamericanas y asiáticas apenas están representadas en el grupo. En 14 de los 32 países incluidos en el conjunto de datos del estudio de elecciones nacionales del CSES, los votantes pueden emitir votos de preferencia para candidatos particulares dentro de un solo grupo de partidos. La elección de preferencia intrapartidaria se proporciona en las elecciones legislativas a la Cámara baja en Bélgica y Chile, o al Parlamento unicameral en la República Checa, Dinamarca, Islandia, Lituania, los Países Bajos, Noruega, Perú, Polonia, Eslovenia y Suecia. Sin embargo, existen variaciones importantes en el método de elección dentro de los partidos en estos países. En algunos contextos, los votantes pueden optar por el voto de preferencia, en otros deben votar por un candidato dentro de una lista de partidos. Por el contrario, en Suiza ${ }^{71}$ y Luxemburgo, los votantes tienen tantos votos como escaños en el distrito electoral. Las variaciones también se reflejan en los diferentes impactos del orden de los candidatos sobre el resultado. En Chile, Polonia, Eslovenia y Suiza y, en la mayoría de los casos, en Dinamarca, el orden electoral dentro de un grupo de partidos está completamente determinado por el número de votos nominativos que reciben los candidatos. En Bélgica, la República Checa, Lituania, los Países Bajos, Noruega ${ }^{72}$ y Suecia, una combinación de pedidos de partidos y votos nominativos determina qué candidatos serán elegidos ${ }^{73}$.

Considerando las diferentes dimensiones analíticas que distinguen los sistemas electorales, es útil clasificar los casos de nuestra muestra ${ }^{74}$. En términos de magnitud de la circunscripción, tenemos suficiente variación dentro de la

70 Marsh, «The Voters Decide?», cit.

71 En esta perspectiva, la papeleta transferible única, así como la papeleta abierta o "panachage» utilizada en Suiza, pueden considerarse casos extremos, más allá de las oportunidades que ofrecen las listas abiertas o el doble voto, ya que permiten que los candidatos sean seleccionados de diferentes partidos y así promover una competencia individual muy alta (dentro del partido) (COLOMER, Handbook of electoral system choice, cit., p. 52).

72 Como presentaré en el párrafo ad hoc sobre el país, el sistema noruego prácticamente funcionaba como un sistema cerrado, aunque en teoría los votantes tienen la opción de marcar nombres de una lista.

73 Finalmente, en otros países como Japón (hasta 1994) y Taiwán, los miembros del Parlamento son elegidos directamente por los votantes. En estos sistemas, los votos solo se otorgan a los candidatos. En los sistemas mayoritarios, como SNTV, también se ha votado con preferencia (Corea del Sur, Japón y Taiwán); en cuanto al «voto transferible» el voto preferencial ha estado vigente en Irlanda desde 1922 (Norris, Electoral Engineering. Voting Rules and Political Behavior, Cambridge, 2004, p. 231).

74 Gallagher and Mitchell, The Politics of Electoral Systems, cit., pp. 8-9. 
muestra. Existe una variación significativa entre los países M. Hay casos en los que solo una circunscripción cubre todo el país, de modo que $M$ es igual al número de parlamentarios que se elegirán en el parlamento. Holanda representa ese caso $(\mathrm{M}=150)$.

De hecho, en este ensayo me he centrado en las formas en que los sistemas de votación preferenciales impactan en la dinámica intrapartidaria. He comenzado el análisis teniendo en cuenta las siguientes preguntas: ¿la votación preferencial realmente confiere más poder a los votantes? ¿Los votantes realmente determinan la elección de un candidato? ¿Es el voto preferencial un sistema que limita severamente las opciones de los votantes? Teniendo en cuenta que los sistemas electorales pueden considerarse como «un conjunto de leyes y normas de partidos que regulan la competencia electoral entre y dentro de los partidos $»^{75}$, era evidente que la dimensión «dentro de los partidos» estaba relativamente poco desarrollada. Esa consideración es cierta, de hecho, Shugart afirmó que «el estudio de la dimensión intrapartidaria se ha visto obstaculizado por una caracterización a veces nebulosa de las variables dependientes, la falta de datos y, lo que es peor, la falta de una comprensión clara de las reglas que se investigan según que países ${ }^{76}$.

Como ha sido extensamente tratado, me he centrado en los sistemas electorales del PLPR y en cuatro subcategorías: lista abierta; lista flexible; listas latentes; sistemas de cuasi-listas. Hacer tal elección me ha permitido investigar mejor las diferencias y similitudes dentro de la familia de los sistemas de votación preferencial. Estos últimos no son «iguales» y difieren tanto en las características como en las consecuencias que producen en los partidos y los resultados electorales. Además, al informar lo que Pedersen indicó en su artículo ${ }^{77}$, es posible hacer una distinción entre el voto preferencial utilizado de manera efectiva y no efectiva.

El enfoque comparativo de este trabajo ha llenado parcialmente la brecha que existía al ofrecer no solo un conjunto de datos, sino también un análisis crucial que prueba diferentes hipótesis. Los resultados son importantes, prometedores y ofrecen muchos puntos de estudio tanto en términos teóricos como empíricos. Tuve la oportunidad de probar la hipótesis relacionada con la pregunta si «realmente los votantes deciden» ${ }^{78}$.

Los principales resultados provienen de los datos sobre cinco variables que potencialmente tienen efectos sobre la rotación de MP. De esta manera, tuve la posibilidad de sopesar el impacto de los votos preferenciales y, en general, de las características de los sistemas de votación preferencial en los candidatos, esa es la

75 Cox, Making votes count, cit., p. 38.

76 SHUGART, «Comparative electoral systems research «, cit., p. 36.

77 Pedersen, «Preferential voting in Denmark: the voters influence on the election of Folketing candidates», Scandinavian Political Studies, vol. 1, 1, 1966, 167-187.

78 Marsh, «The Voters Decide?», cit. 
dinámica intrapartidaria. El objetivo principal era determinar si las preferencias realmente importan en la selección de diputados o si otras variables son más relevantes.

El primer gran hallazgo es que podemos observar tendencias diferenciadas entre los dos grupos principales de sistemas electorales de PLPR, es decir, entre «sistemas de listas flexibles y sistemas de listas latentes» por un lado, y los sistemas de listas abiertas y los sistemas de cuasi listas por otro. A primera vista, los datos han indicado que la variable más importante en los sistemas electorales de listas flexibles y de listas latentes que afectan a la dinámica dentro del partido es el número de cargos reelegidos, mientras que el voto de preferencia representa solo el 6\% . Por lo tanto, los datos sugieren que en estos dos subtipos de PLPR, las preferencias no afectan a la rotación de los cargos, o su influencia es, en el mejor de los casos, una entrada menor. Viceversa, en los sistemas electorales OLPR y de lista latente, el peso de los votos preferenciales no es completamente poco influyente, ya que representa aproximadamente el 13 por ciento de la rotación, aunque es significativamente menos importante que otras variables. Por lo tanto, es bastante claro que las cosas aún suceden entre los partidos, y dentro de las organizaciones del partido, siempre y cuando decidan las candidaturas que afectan el destino del MP más que los votos preferenciales, y mucho más que el poder de los votantes.

Sin embargo, los dos grupos muestran diferencias relevantes en términos de impacto en la dinámica dentro de los partidos. La comparación indica que en tres de cada cuatro casos, los sistemas de cuasi-listas OLPR + funcionan mejor para los países en los grupos de lista flexible y lista latente. El único factor que importa más en los sistemas de listas flexibles + latentes es la reelección, es decir, la proporción de parlamentarios confirmada en las siguientes elecciones. Estos datos son muy importantes, innovadores y no solo tienen relevancia empírica, sino que confirman una consecuencia teórica crucial. Estos apoyan que la votación preferencial sea más importante en OLPR, como es de esperar teóricamente, ya que es mucho más probable que el comportamiento de los votantes influya en la elección de un candidato determinado. Por otro lado, el impacto reducido de los votos preferenciales en los sistemas de listas flexibles subraya la relevancia de los umbrales, que hacen que el sistema electoral funcione casi como un sistema de listas cerradas.

Además, del lado de los partidos políticos, los datos enfatizan que en casos de listas flexibles, la influencia y el poder de las organizaciones del partido son más importantes en la carrera y las reelecciones de parlamentarios que en los sistemas OLPR. La evidencia empírica sugiere que los partidos políticos pueden gestionar un número considerable de resultados de reelección antes de las elecciones a través de su control sobre el orden de clasificación de la lista del partido. Además, en los sistemas de listas flexibles, los umbrales eficientes (en términos de votos personales a ser conquistados) dificultan que los candidatos ubicados en la parte inferior de la lista den forma al orden de la lista del partido y reemplacen a sus compañeros de la parte superior de la lista. 
Además, también surgen diferencias considerando el resultado por país. Los datos enfatizan, una vez más, el papel del sistema electoral, ya que los casos se alinean siguiendo las fronteras de los subtipos de PLPR en lugar de otros factores relacionados con las características de los países. Por lo tanto, es posible resumir que todos los casos ubicados por encima de la media pertenecen al subtipo OLPR. Significativamente, todos los demás países se encuentran por debajo de la media, lo que evidencia una clara división entre los sistemas flexibles y los de lista abierta. Una vez más, desde el punto de vista empírico, los datos indican que el nivel de apertura del sistema de «votación preferencial» depende del poder de los votantes para influir en la selección de candidatos para la elección, y que la presencia de umbrales provoca una diferencia crucial en el giro de la lista flexible sistemas en sistemas funcionalmente CLPR.

Además, cuando tenemos en cuenta únicamente las derrotas y excluimos los parlamentarios que han salido reelegidos, los datos muestran una vez más un patrón bien diferenciado entre los sistemas de listas abiertas y los sistemas de listas flexibles. En el primer caso, la preferencia es importante para un cuarto de todas las derrotas, mientras que en el caso de sistemas electorales «abiertos», el porcentaje alcanza en torno al 55\%. En cambio, en los sistemas de lista abierta, como ya se comentó anteriormente, las preferencias tienen un impacto mucho menor, no solo por la presencia de umbrales legales, sino también probablemente por su magnitud, así como por la decisión de los partidos en las candidaturas y el comportamiento de los votantes.

La regresión de OLS ha evidenciado que el sistema electoral es importante para explicar la variación en el nivel de los parlamentarios salientes que son reelegidos. Esos datos tienen un importante impacto teórico en los efectos de los sistemas electorales, puesto que nos dice que, en presencia de un sistema electoral PLPR, la posibilidad de salir reelegido aumenta parcialmente, pasando de un sistema PLPR menos «abierto» a uno más «abierto», el OLPR.

Resulta interesante observar los efectos de los sistemas electorales PLPR en el número de cargos en funciones que son derrotados por culpa del resultado negativo de un partido. Los valores estandarizados beta nos indican que todos los sistemas PLPR tienen una fuerte y positiva relación con la variable dependiente. Además, el sistema electoral «funciona» muy bien a la hora de explicar las variaciones en la probabilidad de ser derrotado por un sistema electoral PLPR debido al orden de la lista (la variable solo se refiere a los sistemas de lista flexible y a los de lista latente). Esos datos son realmente cruciales desde un punto de vista teórico, puesto que reflejan la importancia de las reglas de los sistemas electorales para conferir más poder a los partidos en detrimento del de los votantes, como normalmente ocurre en los sistemas de lista flexible.

Las probabilidades de ser derrotado por culpa del voto preferencial per se están intensa y positivamente relacionadas con el poder de los votantes, como resultado de las previsiones de los sistemas electorales. La relación entre el sistema de lista «abierta» y su subtipo es fuerte y positiva. Resultan vitales para 
explicar la variación de los efectos de las características de los sistemas electorales en el comportamiento de los votantes y en las dinámicas internas de los partidos, así como las derrotas de los parlamentarios por los votos preferenciales. De este modo, merece la pena mencionar que el poder del votante está positivamente correlacionado con la probabilidad de ser reemplazado por otro candidato por las preferencias, así como el supuesto contrario, el poder de los partidos es un factor correlacionado negativamente con la probabilidad de experimentar una derrota electoral debido a los votos personales. Lo más destacable es que la variable del poder del votante mantiene su fuerza y signo en ambos modelos, confirmando la importancia del poder conferido a los votantes por el sistema electoral. Estos datos respaldan y confirman la pregunta inicial de la investigación y las hipótesis que relacionan las características de los sistemas electorales con los efectos en la competencia dentro de los partidos.

En la misma línea, el impacto de los sistemas PLPR en la probabilidad de que un parlamentario saliente sea reelegido en las próximas elecciones parece ser muy importante.

Por el contrario, si consideramos el efecto que tiene un PLPR en el sistema político, debe mencionarse que las probabilidades de variación en el Número Efectivo de Partidos (NEP) son significativas. Además, el hecho de que el sistema PLPR per se sea significativo representa un nuevo dato de relevancia. Esto último significa que los sistemas electorales, especialmente los de voto preferencial, pueden tener una importante influencia en la estructura del sistema de partidos y la representación. En lo que respecta a la volatilidad electoral, se presume que los PLPR tienen un impacto mayor. Sin embargo, al observar los diferentes «dummies» el OLPR es el único que tiene un valor significativo que explique la probabilidad moderada de volatilidad. Por lo tanto, los datos parecen sugerir que cuando los votantes tienen más poder para elegir a sus candidatos, esta capacidad repercute en nivel general de volatilidad electoral. Esto es una importante evidencia empírica, que respalda la doctrina que aboga por la mayor probabilidad de que los votantes sigan a los candidatos que a los partidos en aquellos sistemas electorales en los que puedan elegir directamente al «caballo» para la carrera.

En lo que respecta al votante, tal y como ha señalado la doctrina, los sistemas PLPR pueden inducir a los ciudadanos a votar más y a emitir una preferencia al aumentar su poder. De hecho, los datos muestran que con que mayor «poder» se les dé a los votantes, más aumentará la probabilidad de que emitan una (o más) preferencia(s). Por el contrario, el índice del partido se correlaciona negativamente con la probabilidad de emitir una preferencia en los sistemas electorales PLPR. Esto es un dato empírico muy significativo y está en la línea de lo dicho por la doctrina, apoyando igualmente el marco teórico de este artículo y la cuestión objeto de investigación, así como la hipótesis. En síntesis, el último resultado es de una gran importancia al afirmar con una prueba empírica que en los sistemas de voto preferencial la probabilidad de emitir una preferencia depende principalmente de las características del sistema PLPR. El nivel de poder 
otorgado a los votantes parece que afecta también a su comportamiento, en términos de la elección del candidato al que apoyan. Considero que esto demuestra que cuando las preferencias de los votantes cuentan para más, estos votos se distribuyen de forma más uniforme entre los candidatos de los partidos. Hasta donde yo sé, esto constituye un descubrimiento original, cuya importancia teórica se puede establecer en referencia al comportamiento del voto. Los datos explican que cuando se otorga poder a los votantes y no están obligados a emitir un voto condicionado, su preferencia se distribuye entre diferentes candidatos y no sólo entre los designados por el partido. No obstante, esta condición per se no es suficiente para provocar más rotación en las siguientes elecciones parlamentarias en los sistemas electorales PLPR.

Esto no es una simple afirmación de lo obvio — «es más probable que los votantes emitan un voto de preferencia cuando estos tienen una buena oportunidad para influir en el resultado que cuando tienen poca o ninguna». Sin embargo, esta suposición nunca ha sido comprobada empíricamente en una perspectiva comparada, por lo que muestra que no es indiferente tener uno u otro sistema PLPR. De hecho, hay variaciones internas entre estos sistemas electorales que provocan diferentes resultados, en términos de competición electoral intrapartidaria, los cuales han sido calculados en este trabajo.

Los datos apuntan a que cuanto más grande es el poder del votante, menor es el índice Gini de votos personales, es decir, una proporción menos concentrada de votos preferenciales en torno a uno o pocos candidatos. En cambio, cuanto mayor es el poder del partido, mayor son los valores del índice Gini, lo que sería una proporción más concentrada de los votos personales en torno a uno o pocos candidatos. Este hallazgo podría interpretarse como la influencia per se de las características de los sistemas electorales en la distribución de votos entre los candidatos, lo que es más relevante que cualquier disposición atribuida a los votantes y/o a los partidos. En resumen, los sistemas PLPR son importantes para contribuir a igualar los votos entre los candidatos de la lista y esta tendencia se fortalece cuando se añaden las disposiciones de los votantes. El poder del votante mantiene su poder explicativo cuando pasa de un modelo simple a un modo en el que todas las variables se tienen en cuenta. Además, en lo que respecta a los supuestos incluidos en la investigación, la tipología basada en el índice de voto preferencial (IPV) evidencia datos de gran interés, que confirman las evidencias empíricas del análisis estadístico. Los países se ubican según el poder del partido y del votante y el análisis estadístico parece respaldar esa distribución especial. Hemos aprendido que el sistema PLPR importa en la rotación parlamentaria entre elecciones. El análisis de la mencionada tipología, la cual ha sido desarrollada en base a los índices estandarizados de votantes y de partidos políticos, ha evidenciado claramente la existencia de algunos parámetros. Además, el análisis estadístico también ha demostrado la importancia de las disposiciones de los sistemas electorales. Como la regresión lineal ha subrayado, el factor índice de votante es casi siempre estadísticamente significativo. Por último, he calculado la 
correlación entre una característica crucial de un sistema electoral, la del poder del votante, y dos variables indispensables para entender mejor los efectos del voto preferencial y para comprobar más a fondo sus efectos. La relación entre los poderes del votante y la abstención es especialmente fuerte, significativa según las estadísticas, pero negativa. Ello constituye un resultado empírico y comparativo de gran importancia que arroja luz sobre las familias de los sistemas PLPR, aunque se pueda hacer un mayor análisis a nivel de estudio de casos o, como ya se dijo, considerando también la participación como dependiente de otros factores o incluso considerando al PV como una variable dependiente. De manera análoga, los datos indican que el poder del votante y el porcentaje de votos preferenciales emitidos es fuerte, significativo y positivo. De este modo, al igual que en el caso de la participación, estos datos amplían nuestro conocimiento en los efectos de los sistemas electorales preferenciales en el comportamiento del votante, los sistemas electorales importan. No sólo los sistemas PV dan forma a las dinámicas internas de los partidos y a los resultados, sino que también surgen diferencias importantes cuando se tienen en cuenta los diferentes subtipos de PLPR y sus características. Las principales hipótesis se ven así confirmadas (por ejemplo, el nivel de derrotas de los cargos en funciones aumenta al pasar del sistema PLPR menos «abierto» a un sistema más «abierto», como el caso del OLPR; los votantes tienden a utilizar su poder cuando tienen derecho a expresar su voluntad en términos electorales; el número efectivo de partidos aumenta en función de los poderes de los votantes y el nivel de apertura de los sistemas electorales; los sistemas PLPR, en diferente grado dependiendo de la apertura de los subtipos, muestran los altos niveles de volatilidad electoral; los datos, tanto de regresión como de correlación, nos indican que con que mayor es el poder de los votantes en emitir una preferencia, menor es su abstención).

Los resultados arrojan luz no sólo en el lado empírico del campo electoral, sino que también subrayan las consecuencias teóricas de adoptar un concreto sistema electoral, especialmente el preferencial, las cuales serán críticas tanto para los académicos como para los políticos, los partidos y los votantes. El análisis estadístico ha probado importantes hipótesis relacionadas con el impacto de los sistemas electorales (PLPR) en las dinámicas intrapartidarias, lo que ha sido investigado relativamente poco hasta ahora. Los datos representan un importante paso adelante tanto en términos de la teoría sobre el impacto de los sistemas electorales, como en la medición de dicha relación entre los sistemas electorales (PLPR) y las dinámicas intrapartidarias.

TITLE: Preferential voting systems. effects on intra-party competition and voting behavior

ABSTRACT: This article examines the effects of preferential voting on intraparty electoral competition and voting behavior. Using data covering 19 countries and over 200 elections, this study sheds light on a somewhat neglected aspect of electoral systems. The author demonstrates that the ability of voters to influence 
the selection and deselection of MPs under preferential voting systems is not as important as is often assumed. Instead, their ability to shape the election of a given candidate depends beavily on the balance between party power and voter power. In this way, this work advances the understanding of the effect of preferential voting on intra-party dynamics, parliamentary turnover, and voter behavior.

RESUMEN: Este artículo examina los efectos del voto preferencial en la competencia electoral intrapartidaria y en el comportamiento del voto. Mediante los datos recogidos en 19 países y más de 200 elecciones, este estudio arroja luz en un aspecto ciertamente olvidado de los sistemas electorales. El autor demuestra que la capacidad de los votantes de influir en la selección y el descarte de parlamentarios en los sistemas de voto preferencial no es tan importante como se suele señalar. En cambio, su capacidad para dar forma a la elección de un determinado candidato depende enormemente del equilibrio entre poder de partido y poder del votante. De esta manera, este trabajo avanza en la compresión del efecto del voto preferencial en las dinámicas intrapartidarias, en la rotación parlamentaria y en el comportamiento del votante.

KEY WORDS: Preferential voting; electoral systems; electoral behavior; Intra-party competition; parliamentary turnover

PALABRAS CLAVE: Voto preferencial; sistemas electorales; comportamiento electoral; competencia intrapartidaria; rotación parlamentaria

FECHA DE RECEPCIÓN: 12.01.2020

FECHA DE ACEPTACIÓN: 29.02.2020 
